\title{
Sensibilización a las lenguas: una propuesta didáctica en las primarias de Guadalajara.
}

\section{Language awareness: a didactical proposal in Guadalajara primary schools.}

\author{
Anne-Catherine Didier \\ Departamento de Lenguas Modernas / Universidad de Guadalajara (MÉXICO) \\ CE: acdidier3@yahoo.com ID ORCID: 0000-0002-9114-600X
}

DOI: $10.32870 /$ sincronia.axxiii.n76.35b19

(C) $\mathrm{BY} \cdot \mathrm{NC}$

Esta obra está bajo una Licencia Creative Commons Atribución-NoComercial 4.0 Internacional

Recibido: $26 / 11 / 2018$

Revisado: $19 / 12 / 2018$

Aprobado: 09/05/2019

\section{RESUMEN}

Frente a la demanda creciente de clases de lenguas extranjeras en Guadalajara para el público infantil, es decir en kínder o primaria, la Licenciatura en Didáctica del Francés como Lengua Extranjera del departamento de lenguas modernas de la Universidad de Guadalajara inicio un programa de prácticas profesionales con este público. Este artículo relata la aplicación de una propuesta didáctica llamada despertar o sensibilización a las lenguas en algunas primarias de Guadalajara. Primero, se describe el contexto en el cual fue llevado a cabo este proyecto. Luego, se explica en que consiste el despertar o sensibilización a las lenguas. En una tercera parte, se presenta la propuesta didáctica aplicada para esta experiencia y finalmente, se presentan los resultados del análisis.

Palabras clave: Sensibilización a las lenguas. Francés precoz. Niño. Evlan. 


\begin{abstract}
:
In front of the increasing demand in Guadalajara of foreign languages classes for children, in kindergarden or primary school, the BA of Didactic of French as a Foreign Language of the Modern Language Department of the University of Guadalajara began a professional practices program with this public. This article relates the application of a didactical approach called language awareness in some primary schools of Guadalajara. First, we describe the context in which this research took place. Then, we explain what language awareness is. In a third part, we present the didactical proposal applied for this experience and finally, we present the analysis results.
\end{abstract}

Keywords: Language awareness. Early French learning. Child. Evlang.

\title{
Intrpducción
}

Hoy en día, las instituciones que ofertan clases de idioma para niños son cada vez más numerosas en México y en Guadalajara en particular. Sin embargo, por lo general, no son los niños quienes deciden aprender un idioma extranjero sino sus papas o las instituciones. Así que se plantea la cuestión de cómo motivarlos a aprender una lengua. Frente a esta interrogante, pensamos que la propuesta didáctica llamada despertar o sensibilización a las lenguas (EVL por sus siglas en francés: Eveil aux langues) puede ser una respuesta. ¿En qué consiste? ¿Cuáles son sus objetivos? ¿Cuál es su metodología? Este enfoque es muy amplio y es muy fácil perderse en sus numerosas aplicaciones pedagógicas así que nos enfocamos en tratar de proponer un instrumento para delimitar de manera precisa los objetivos, documentos y actividades que se van a elaborar.

En este artículo, después de describir el contexto en el cual se desarrolló este proyecto, explicaremos en una segunda parte los lineamientos del despertar a las lenguas, luego describiremos la propuesta didáctica implementada en algunas primarias públicas de Guadalajara, y terminaremos con un análisis de su aplicación. 


\section{Contextualización del proyecto}

En 2016, la Dirección de Educación Municipal de la Secretaría de Educación de Jalisco (SEJ) inició un nuevo programa titulado Vinculación Para La Innovación Educativa que consistía en impartir clases de francés en algunas escuelas primarias públicas de Guadalajara. Para llevar a cabo este proyecto, solicitó el apoyo de la Licenciatura en Didáctica del Francés como Lenguas extranjera (LIDIFLE) del Departamento de Lenguas Modernas (DELEM) de la Universidad de Guadalajara. A fin de responder a esta demanda, la LIDIFLE puso en marcha un programa piloto en el marco de la clase de Micro Enseñanza IV en el cual los estudiantes de octavo semestre realizarían sus prácticas profesionales como maestros de francés con un público de niños. Sin embargo, frente a la urgencia, los maestros practicantes no contaban con una preparación suficiente para este público porque solo habían realizado sus prácticas con adultos. Ante esta situación se realizaron unos ajustes dentro del plan de estudio de la licenciatura empezando por cambiar la materia de Francés para niños en séptimo semestre en lugar de noveno semestre para que los estudiantes de la siguiente generación tuvieran una formación para impartir clases a niños antes de sus prácticas de octavo semestre.

En 2017, con la siguiente generación de practicantes, que contaba ya con una formación para enseñar a niños, se pudo dar seguimiento al proyecto de manera más formal. En el marco de la clase de Micro Enseñanza IV y del programa PAL (Programa Abierto de Lenguas), se abrieron 13 grupos de 18 a 35 niños de sexto año de primaria en nueve escuelas primarias públicas de Guadalajara con 20 profesores practicantes de octavo semestre. Se impartió 1 h30 por semana repartida en 45 minutos los martes y 45 minutos los jueves. Las prácticas duraron 24 horas entre el mes de febrero y el mes de mayo. Los maestros en formación desarrollaron dos unidades de aprendizaje: una unidad de EVL y una unidad de francés de 12 horas cada una.

Se decidió iniciar las clases con el enfoque de EVL (Eveil aux Langues) es decir de despertar o sensibilización a las lenguas antes de empezar con el aprendizaje del francés a fin de desarrollar la motivación de los niños para aprender un idioma extranjero. En efecto, ellos no decidieron aprender francés sino que fue decisión de la institución. Para desarrollar su interés frente a una realidad tan distante y distinta a la suya nos pareció fundamental empezar con esta sensibilización. 


\section{Despertar a las lenguas (EVL)}

EL despertar a las lenguas inició en los años 1970 en Gran Bretaña con los trabajos de Erik Hawkins (Language awareness) a los cuales Michel Candelier y Louise Dabene dieron seguimiento en los años 1980 en Francia. Candelier (2011) nos recuerda que la finalidad de este enfoque es "contribuir a la construcción de sociedades solidarias, lingüística y culturalmente pluralistas". Para lograrlo es necesario desarrollar dos competencias principales: la competencia a aprender lenguas extranjeras y la competencia a vivir en una sociedad plurilingüe y pluricultural por medio de saberes, saberhacer y saber-ser tales como: la apertura a la diversidad lingüística y cultural así como la motivación para el aprendizaje de lenguas; aptitudes de orden metalingüístico/metacomunicativo como la observación y el razonamiento y de orden cognitivo facilitando el acceso al dominio de las lenguas; un conjunto de referencias que ayudan a la comprensión del mundo plurilingüe y pluricultural. Para más detalles, se puede consultar el referencial de objetivos en internet (Candelier, 2001). Las unidades de aprendizaje de despertar a las lenguas se imparten entonces en lengua materna (en nuestro caso en español) y consisten en actividades basadas en la observación, la reflexión y el análisis contrastivo y comparativo entre la lengua materna y otras lenguas. Se trata de lograr una descentración lingüística y cultural a fin de desarrollar actitudes positivas y de tolerancia hacia el otro y disminuir los estereotipos. Se trata de desarrollar en el niño un conjunto de competencias pre-lingüísticas y permitirle abrirse a la diferencia, al otro y tomar un poco de distancia con su propia lengua-cultura, de permitirle "despertar su consciencia metalingüística, de tomar consciencia de lo arbitrario de las lenguas y culturas gracias a la descentración y distanciación" (Cuq y Gruca, 2005 , p.357). Por lo tanto, el objetivo de este enfoque no es aprender una lengua extranjera sino preparar los alumnos al aprendizaje de lenguas extranjeras. En otras palabras, se trata de "un verdadero propedéutico al plurilingüismo, al intercultural y a la reflexión metalingüística, y un fortalecimiento por contraste de la lengua materna" (Cuq y Gruca, 2005, p.358).

Para llevar a cabo nuestro proyecto nos basamos en el programa Evlang. Se trata de un programa europeo para las escuelas primarias que se llevó a cabo entre 1997 y 2001 auspiciado por 
la Comisión europea (Lingua action D) con la participación de Austria, España, Italia, Suiza y Francia. Este programa consistió en producir material didáctico para clases de francés como Lengua Extranjera (FLE) a niños de cuarto y quinto año de primaria, formar profesores y evaluar estas clases. Evlang está conformado por 12 dominios, módulos o unidades de aprendizaje que son los siguientes:

1. Las relaciones entre las lenguas et las culturas.

2. El objetivo consiste en que los alumnos tomen consciencia que el mundo se percibe a través de la lengua y cultura.

3. Las relaciones entre las lenguas: historia y evolución de las lenguas.

4. Se trata de que los alumnos descubran que existen familias de lenguas y que sus similitudes pueden ayudar a la intercomprensión. También se ve la evolución de las lenguas en la historia y las migraciones.

5. No se escribe como se habla y no se habla como se escribe.

6. Consiste en hacer descubrir a los niños que existen un código oral y un código escrito y que el funcionamiento de cada uno es distinto.

7. Lo verbal y no-verbal.

8. Que los alumnos tomen consciencia que existen otros sistemas de comunicación (kinestésica, prosémica) que la verbalización es el objetivo central de este campo temático.

9. Los sistemas de escritura, sonido y grafía.

10. Consiste en que los niños se den cuenta que existen diferentes sistemas de escritura (alfabético, jeroglífico, ideográmico) con principios diferentes y que la relación sonido-grafía puede ser distinta según la lengua.

11. Las regularidades en la lengua (morfosintaxis, léxico, texto...).

12. Se trata de que los alumnos observen y comparen la estructura de las lenguas. Por ejemplo, que descubran que existen lenguas aglutinantes, o de cómo se forman los adverbios o adjetivos en una lengua y en otra.

13. La variación (neologismos). 
14. Este módulo consiste en que los alumnos tomen consciencia de que las lenguas se "prestan » palabras y que es una riqueza.

15. Los sistemas fonológicos (el mundo sonoro de las lenguas).

16. El objetivo de esta unidad es que los niños comparen los fonemas y descubran la gran diversidad de medios sonoros de las lenguas (clics, tonos, etc.). Se aborda también la prosodia.

17. Las lenguas en el espacio: el niño y las lenguas, el entorno, Europa, el mundo...

18. En este módulo, se trata de que los alumnos tomen consciencia de que las lenguas y culturas están presentes en su entorno y que son parte de su socialización. También descubren la presencia de las diferentes lenguas en el mundo.

19. El bilingüismo y la diglosia (registros y variedades de lenguas, plurilingüismo).

20. El objetivo consiste aquí en que los niños descubran que existen variaciones (ligada a la historia, a la situación de comunicación, al estatus de las lenguas y de las personas) dentro de una lengua y entre diferentes lenguas.

21. El estatus de las lenguas.

22. Esta unidad busca concientizar a los alumnos de que se atribuye un estatus más o menos favorable a las lenguas y que los locutores que hablan estas lenguas están categorizados y jerarquizados. Se pueden estudiar los conceptos de lengua y dialecto por ejemplo.

23. La apropiación de las lenguas: adquisición y aprendizaje.

24. El objetivo es que los alumnos reflexionen sobre cómo se aprende una lengua diferente a la suya. (Candelier, 2011 ; Kervran \& Candelier, 2003).

Como lo dice Candelier, el despertar a las lenguas no pretende hacer de los niños "unos lingüistas precoces" ni unos historiadores precoces; cada módulo busca concientizar a los niños sobre un tema específico.

Como se puede ver, el despertar o la sensibilización a las lenguas es una propuesta muy amplia y contempla una inmensa posibilidad de temas y actividades. A fin de canalizar estas 
opciones y organizarlas, delimitarlas, se propuso un instrumento pedagógico para establecer objetivos precisos.

\section{Propuesta didáctica}

A fin de ayudar a los maestros practicantes a no perderse en este mar de posibilidades, se les dio una ficha técnica (Documento 1) que tuvieron que llenar antes de elaborar su unidad de EVL. Esta ficha técnica incluye cuatro rubros. El primero, dominio/objetivo global consiste en escoger uno de los 12 módulos del programa Evlang que acabamos de mencionar como objetivo global de la unidad. La mayoría de los practicantes escogieron el primer o segundo módulo. En el segundo rubro, objetivos, tuvieron que definir los objetivos específicos de la unidad, es decir los saber ser, saber hacer y saberes relativos a la competencia "aprender las lenguas" y a la competencia "vivir en une sociedad plurilingüe y pluricultural". Se apoyaron en el referencial de objetivos propuestos por Candelier (2001) para llenar este rubro. Algunos ejemplos de estos objetivos específicos son: “aceptación positiva de/interés hacia la diversidad lingüística (y cultural) y los objetos que la constituyen"; o "valorización de las personas bilingües/plurilingües, independientemente del estatus de las lenguas que dominan"; o "distinguir diferentes esquemas entonativos y rítmicos"; o "saber identificar algunas lenguas no conocidas"; o "saber que existen entre las lenguas tanto diferencias como similitudes"; o "saber que existe una gran pluralidad de lenguas a través del mundo y que seguido existen varias lenguas en un mismo país o la misma lengua en varios países", etc. En el tercer rubro, relación programas SEP, tenían que relacionar su unidad con algunos programas y contenidos de la SEP a fin de poder apoyarse sobre algunos conocimientos previos de los alumnos. En efecto, se trata de integrar el EVL a la currícula y no concebirlo como una materia desarticulada de las demás. En el cuarto rubro, características de los niños, los profesores en formación tenían que precisar las características del desarrollo de los niños de 10-12 años según los diferentes estadios de Piaget y Erikson entre otros autores, a fin de proponer en la unidad actividades y documentos adaptados a este público. 




\section{Documento 1}

Anne-Catherine, Didier (2017) Elaborado a partir de los trabajos de Candelier

Después de completar la ficha técnica, los practicantes elaboraron su unidad de EVL a partir de un esquema metodológico propuesto por la Secretaría de Educación Pública del gobierno del GrandDuché de Luxemburgo (2008, pp.16-17). Escogimos este esquema porque define claramente tres etapas que ayudan a organizar la progresión de las actividades de EVL: 1) la puesta en situación, 2) la situación búsqueda y 3) la síntesis.

La puesta en situación consiste en actividades cuyo objetivo es "dar sentido a la actividad integrándola en situaciones de aprendizaje actual, en la vida cotidiana del salón y en el programa 
[para] hacer emerger un verdadero cuestionamiento" (op. cit.). Se trata de despertar el interés de los niños sobre el tema así como de acercarlo a su entorno.

En seguida, se muestran dos ejemplos de actividades de esta primera etapa (Documento 2 y Documento 3) elaboradas por dos maestros practicantes:

Actividad 1: Mi red de lenguas

Tiempo previsto: 20 minutos

Consigna: ¿Cuáles tu relación con las lenguas?

Escribe en cada hexágono (en cada área de tu entorno: familia, amigos, películas, libros, programa de televisión, música, intemet, etc.)) cuál es tu contacto con las lenguas. Por ejemplo, si tienes familiares que hablan otro idioma, si conoces otro idioma en una caricatura, una pelicula, etc.



Actividad elaborada por Fernanda y Joshua

\section{Documento 2}

Actividad elaborada por Fernanda y Joshua 


\section{Actividad 2: La cebolla de preguntas}

Tiempo previsto: 10 minutos

Consigna:

Avienta la cebolla y desdobla cada capa. Lee las preguntas escritas a dentro en voz alta y contesta. Si no sabes la respuesta, tus compañeros pueden contestar. Después pueden comentar las respuestas.

Las preguntas escritas en la pelota-cebolla:

1. ¿Has visto programas en la televisión, caricaturas o videos que estén en otra lengua que no sea el español?

2. ¿Conoces alguna lengua que se escriba de izquierda a derecha?

3. ¿Sabes qué significa Как поживаете? ¿Sabes en que lengua está escrito?

4. ¿Conoces al menos tres paises en los que se hable el portugués?

5. ¿Conoces al menos cuatro países en los que se hable en español?

6. ¿Cuántas lenguas oficiales hay en México?

7. ¿Sabes decir "gracias" en al menos 3 lenguas diferentes?

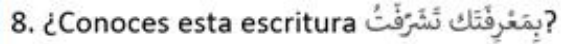

9. ¿Has oído a alguien hablar francés?

10. ¿Sabes contar en al menos 3 lenguas?

11. ¿Conoces personas que hablen italiano?

12. ¿Qué significa para ti la diversidad lingüística?

13. ¿Conoces a alguien que hable más de 3 lenguas?

14. ¿Has oído a alguien hablar chino?

15. ¿Has oído a alguien hablar alemán?

16. ¿Has escuchado alguna canción en otro idioma que no sea el español?

17. ¿Sabes cuál es el origen de tu nombre?

18. ¿Conoces a alguien con un nombre de origen náhuatl? ¿Sabes su significado?

19. ¿Conoces palabras de origen árabe?

20. ¿Qué es una lengua?

21. ¿Cuál es la diferencia entre una lengua y un dialecto?

22. ¿Cuál es la diferencia entre lengua e idioma?

23. ¿En qué paises del mundo se habla francés?

\section{Documento 3}

Creado por Fernanda y Joshua; preguntas tomadas en su mayoría de ELODIL (2013) 
La situación búsqueda comprende actividades que buscan la "apropiación de nuevos conocimientos". Es el núcleo de la unidad, etapa durante la cual los alumnos van a intentar resolver problemas gracias a la observación, la clasificación, el análisis, la comparación, etc. entre diferentes lenguas-culturas y la propia. Es una fase de exploración y conceptualización donde los niños tienen que descentrarse.

A continuación, se da un ejemplo de actividad de esta segunda etapa (Documento 4):

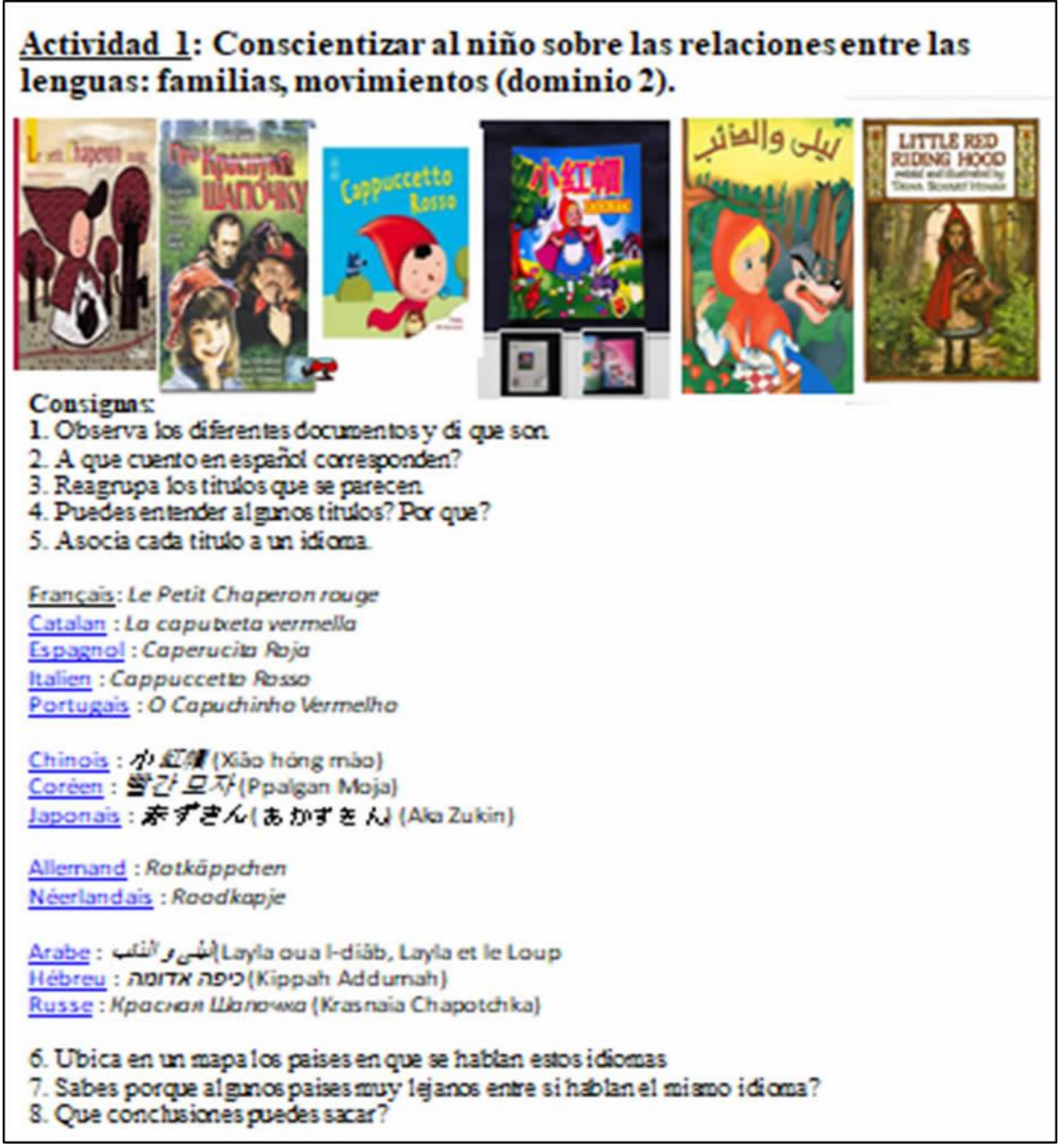

\section{Documento 4}

Modificado por Anne-Catherine Didier (2017), a partir de las propuestas de Dabene (1991, p. 63) y de Candelier, Kervan (2003). 
Finalmente, la síntesis permite la "consolidación de los aprendizajes y la concientización de los saberes adquiridos y por adquirir" al realizar productos concretos de los principales descubrimientos. En esta última etapa, los alumnos hacen un balance de sus aprendizajes.

Abajo, se propone un ejemplo de actividad de esta tercera etapa (Documento 5):

\section{Actividad 1: Portafolio}

Tiempo previsto: 40 minutos

\section{Consigna:}

Por equipo de 3 , van a plasmar en una cartulina todo lo que han aprendido a lo largo de la unidad. Organicen las informaciones por país, por tema con lo que más les gusto o sorprendió. Pueden dibujar banderas, escribir palabras, saludos en otros idiomas, etc.| Luego, cada equipo va a presentar su cartulina al grupo.

\section{Documento 5}

Elaborado por Fernanda y Joshua

Mediante estas tres etapas, los practicantes elaboraron sus unidades de EVL.

\section{Análisis de resultados}

La metodología está fundamentada en una investigación cualitativa a base de observación en la cual buscamos apreciar el impacto del EVL sobre dos aspectos.

En un primer tiempo, quisimos analizar la motivación de los niños, la cual representaba nuestra primera preocupación. Las dos hipótesis tomadas de Kervran \& Candelier (2003) eran que el EVL permitiera 1) hacer evolucionar las representaciones y actitudes de los alumnos acerca de las lenguas y culturas con una mayor apertura e interés hacia lo diferente y la diversidad; 2) desarrollar su deseo de aprender idiomas. A fin de verificar estas hipótesis, elaboramos tres cuestionarios aplicados al final de la unidad de EVL: el primero a 200 niños, el segundo a los 20 practicantes y el tercero a los dos maestros observadores. 
Los resultados detallados de esta primera investigación se encuentran en un previo artículo (Didier, 2018) del cual podemos resumir la información de la manera siguiente. Después de esta unidad de EVL, los niños demostraron que estaban muy motivados por aprender una lengua extranjera y descubrir otras culturas. Les gustaron mucho las actividades de EVL. Este entusiasmo general se confirmó con las respuestas de los profesores practicantes y de los observadores a través de las cuales destacaron una evolución positiva de las representaciones de los niños hacia el otro, más en el plano afectivo que cognitivo por la brevedad del tiempo dedicado al EVL. Un maestro comenta por ejemplo que algunos niños iniciaron el EVL con muy poco interés hacia el aprendizaje de idiomas y pensaban que era "tonto" aprender una lengua y que terminaron la unidad con el deseo de aprender muchas lenguas y con otra visón del mundo.

En un segundo tiempo, para la investigación actual, quisimos analizar la pertinencia de la propuesta didáctica. Elaboramos otro cuestionario aplicado a los practicantes para conocer la operacionalidad del instrumento (ficha técnica y organización de la unidad en tres etapas) así como una rejilla de observación que llenaban los profesores observadores durante cada clase para triangular la información dada por los practicantes. De estos instrumentos se obtuvo la información siguiente.

Todos los practicantes piensan que la propuesta didáctica de EVL es interesante y el 75\% de ellos la califican útil. Sin embargo, el $66 \%$ la consideran difícil de desarrollar en particular para la determinación de los objetivos.

Si bien la mayoría de los profesores (3/4) afirman que les pareció evidente distinguir los objetivos y actividades entre las tres etapas de la unidad (puesta en situación, situación investigación y síntesis), los observadores por su parte notaron que en algunas ocasiones los practicantes confundían los objetivos de las diferentes etapas y proponían actividades que correspondían a otra etapa. Destacaron que a veces, no se veía una real progresión entre las actividades de las tres etapas y que los practicantes no entendían bien la diferencia de objetivos entre unas y las otras lo que, en algunas ocasiones, hacía un poco repetitivas las actividades. 
La etapa que les costó más problemas a los profesores en formación fue la segunda (situación investigación) porque se les dificultaba encontrar documentos. Mientras que la etapa que les pareció más fácil fue la primara (puesta en situación) porque se basa en los conocimientos previos de los alumnos. Este resultado se confirma por los observadores que indicaron que la etapa de situación investigación no era lo suficientemente desarrollada; que las actividades propuestas por los maestros se limitaban a la observación y no proponían bastantes actividades de razonamiento para los niños. Entonces, para los observadores esta etapa de conceptualización era incompleta.

En la pregunta 6, los practicantes tenían que clasificar cinco afirmaciones por orden de importancia sobre lo que las diferentes etapas permitieron desarrollar con los niños. Sumando todos los resultados, para los practicantes estas etapas favorecieron principalmente la verbalización de los descubrimientos, luego la emisión de hipótesis, de ponerlas a discusión y de compartirlas. Y lo que menos desarrollaron fue tomar consciencia de sus recursos lingüísticos.

A todos los practicantes les pareció difícil encontrar documentos. De hecho, la dificultad de encontrar documentos es un tema recurrente en el cuestionario. Preocupación que tenemos que tomar en cuenta para integrarla a la formación.

En la pregunta 8, los practicantes tenían otra vez que clasificar por orden de importancia siete objetivos que lograron las actividades de EVL. Según ellos, las actividades de EVL primero hicieron evolucionar las representaciones y actitudes de los alumnos acerca de las lenguas y culturas en el sentido de una mayor apertura a lo desconocido y de un mayor interés hacia la diversidad; luego, contribuyeron a una valorización de cada lengua y cultura para los alumnos de orígenes lingüísticos y culturales diversos. Mientras que para ellos, las actividades favorecieron menos la concientización y el desarrollo del dominio de algunas estrategias de aprendizaje lingüístico y las competencias de los alumnos en los aprendizajes lingüísticos en clase o posteriormente.

Finalmente, la mayoría de los practicantes (3/4) piensa que el EVL es esencial antes de empezar una lengua extranjera, importante para introducir los niños a las lenguas, permitió a los niños estar preparados, que todo el mundo debería usar EVL y fue más fácil porque no se abordó la 
lengua extranjera directamente. Un practicante contesta que el EVL restó tiempo al aprendizaje de francés y que un mes de EVL fue demasiado largo. Sin embargo, reconoce que fue interesante y divertido para los niños.

\section{Conclusión}

Para concluir podemos decir que estamos conscientes de los límites de esta investigación porque una sola unidad de EVL es muy poco para modificar las representaciones de los niños y prepararlos más al aprendizaje de una lengua. Es un proceso que se desarrolla a largo plazo y se necesitaría de un estudio longitudinal. Sin embargo, a raíz de estos resultados, podemos afirmar que estas actividades de EVL permitieron una evolución positiva de las representaciones y actitudes de los niños acerca de las lenguas y culturas en el sentido de una mayor apertura a lo desconocido y de un interés más grande hacia la diversidad, y que los primeros resultados fueron positivos en cuestión a su motivación y deseo para aprender una lengua extranjera.

Por otro lado, si bien es cierto que la propuesta didáctica es aceptada y considerada por los practicantes como interesante, necesaria, útil y pertinente, y que tanto la ficha técnica como el esquema metodológico aplicado a la unidad de EVL, ayudaron a los maestros a delimitar sus objetivos y organizar sus unidades, cabe destacar sin embargo que es necesario mejorar la formación de los maestros en tres puntos principales para llevar a cabo esta propuesta de manera más operacional. Primero, enfatizar la formación en el marco de la materia de Francés para niños sobre la distinción entre los objetivos de las tres etapas de la unidad. Segundo, desarrollar más actividades de búsqueda de documentos pertinentes en relación a los objetivos y adecuados para este público. Tercero, trabajar más la segunda etapa para que sea realmente una fase de conceptualización.

Finalmente, es importante mencionar que necesitamos la oportunidad de ampliar el tiempo de prácticas con el público infantil a fin de poder a la vez desarrollar más la unidad de EVL sin restarle tiempo a la enseñanza de la lengua. 


\section{Referencias}

Candelier, M. (2001, actualizado el 15 abril 2011). “Les démarches d'éveil à la diversité linguistique et culturelle dans l'enseignement primaire". En actas del seminario L'enseignement des langues vivantes, perspectives, 27 y 28 marzo 2001, Ministère de l'Education nationale, Direction de l'Enseignement scolaire. Recuperado de http://eduscol.education.fr/cid46536/les-demarches-d-eveil-a-la-diversite-linguistique-etculturelle-dans-l-enseignement-primaire.html

Cuq, J.-P, Gruca, I. (2005). Cours de didactique du français langue étrangère et seconde. Grenoble: PUG.

Gouvernement du Grand-Duché de Luxembourg (2008). Ouverture aux langues à l'école, Luxwmburgo : Ministère de l'Education Nationale et de la Formation professionnelle, Wessen-Kennen-Wellen Plan d'action langues. Recuperado de http://www.men.public.lu/catalogue-publications/themes-pedagogiques/enseignementlangues/langues-ecole/fr.pdf.

Didier, A.-C. (2018). Éveil aux langues pour les enfants : un projet en construction dans les écoles primaires de Guadalajara in Synergies Mexique No.8

Kervran, M., Candelier, M. (2003). “Les activités EVLANG : tâches, objectifs et domaines. L'éveil aux langues à l'école primaire. Evlang : bilan d'une innovation européenne": de Boeck Supérieur. Recuperado de http://jaling.ecml.at/pdfdocs/contributions/candelier kervran.pdf 